\title{
Spinning black holes for generalized scalar-tensor theories in three dimensions
}

\author{
Olaf Baake๑* \\ Instituto de Matemática y Física, Universidad de Talca, Casilla 747, Talca 3460000, Chile, \\ and Centro de Estudios Cientificos (CECs), Avenida Arturo Part 514, Valdivia 5090000, Chile \\ Moisés Bravo-Gaete $\oplus^{\dagger}$ \\ Facultad de Ciencias Básicas, Universidad Católica del Maule, Casilla 617, Talca 3460000, Chile \\ Mokhtar Hassaine \\ Instituto de Matemática y Física, Universidad de Talca, Casilla 747, Talca 3460000, Chile
}

(Received 31 May 2020; accepted 9 July 2020; published 30 July 2020)

\begin{abstract}
We consider a general class of scalar tensor theories in three dimensions whose action contains up to second-order derivatives of the scalar field with coupling functions that only depend on the standard kinetic term of the scalar field, thus ensuring the invariance under the constant shift of the scalar field. For this model, we show that the field equations for a stationary metric ansatz together with a purely radial scalar field can be fully integrated. The kinetic term of the scalar field solution is shown to satisfy an algebraic relation depending only on the coupling functions, and hence is constant while the metric solution is nothing but a BañadosTeitelboim-Zanelli (BTZ)-like metric with an effective cosmological constant fixed in terms of the coupling functions. As a direct consequence the thermodynamics of the solution is shown to be identical to a BTZ-like one with an effective cosmological constant, despite the presence of a scalar field. Finally, the expression of the semiclassical entropy of this solution is also confirmed through a generalized Cardy-like formula involving the mass of the scalar soliton obtained from the black hole by means of a double Wick rotation.
\end{abstract}

DOI: $10.1103 /$ PhysRevD.102.024088

\section{INTRODUCTION}

Since the discovery of the Bañados-Teitelboim-Zanelli (BTZ) black hole solution [1], the study of three-dimensional gravity has received considerable attention to such an extent that it is now considered an interesting laboratory to explore the many facets of the lower-dimensional physics at the classical level but also at the quantum level. By threedimensional gravity we are referring not only to Einstein's standard action but to all of its possible variations, including, for example, its higher-order massive theories, such as the topologically massive gravity [2], or the new massive gravity [3]. The three-dimensional gravity models, with or without matter source, are likewise of importance due to the variety of their solutions, and particularly their asymptotic AdS black hole solutions whose near horizon geometry can be

\footnotetext{
*olaf.baake-at-inst-mat.utalca.cl

'mbravo-at-ucm.cl

"hassaine-at-inst-mat.utalca.cl
}

Published by the American Physical Society under the terms of the Creative Commons Attribution 4.0 International license. Further distribution of this work must maintain attribution to the author(s) and the published article's title, journal citation, and DOI. Funded by SCOAP. relevant to test some conceptual aspects of the AdS/CFT correspondence $[4,5]$. In this aspect, the BTZ solution is of particular interest because its in-depth study over the past three decades has considerably enhanced our knowledge on the statistical interpretation of the black hole entropy, see e.g., [6-8]. It is further fascinating that BTZ-like metrics arise as solutions of radically different three-dimensional gravity models. To illustrate this statement, we could mention for example the emergence of BTZ-like solutions in the context of massive gravity [9], in higher-order theories [10], but also in the presence of matter source, such as a scalar (dilatonic) field, see e.g., [11,12]. In the present work, we will confirm this trend by showing that the equations of motion of a general class of scalar tensor theories, enjoying a shift symmetry of the scalar field, and involving up to second-order derivatives of the scalar field, can be fully integrated and solved by a BTZ-like metric.

The interests of studying scalar tensor theories is mainly due to the fact that it constitutes one of the simplest modified gravity theories by extending general relativity with one or more scalar degrees of freedom. The dedication to scalar theories is not new and its origin may be attributed a posteriori to the seminal work of Horndeski [13], who presented the most general scalar tensor theory in four 
dimensions with second order equations of motion. The requirement not to have more than two derivatives in the equations of motion is connected to the Ostrogradski theorem which states that (under certain assumptions) higher-order derivative theories have a Hamiltonian that is unbounded from below. This is related to the appearance of an extra (ghost) degree of freedom with negative energy. Thus the absence of higher time derivatives in the equations of motion guarantees the absence of the Ostrogradski ghost. Nevertheless, it has been shown recently that some particular higher-order theories of a single scalar field extension of general relativity can propagate healthy degrees of freedom and are mechanically stable. The most general such Lagrangian depending quadratically on second-order derivatives of a scalar field was constructed in [14,15], and dubbed degenerate higher order scalar tensor (DHOST) theory. This terminology indicates that the absence of Ostrogradski ghosts is mainly due to the degeneracy property of its Lagrangian. There even exists a subclass of DHOST theories where gravitational waves propagate at the speed of light, being in perfect agreement with the observed results [16]. While these attractive properties of scalar tensor theories occur in four dimensions, we nevertheless like to explore the implications of such models in three dimensions. This is precisely the aim of the present work.

Here, we will consider a general scalar tensor theory in three dimensions with a field content given by the metric $g$ and a scalar field denoted by $\phi$. The main assumption concerning the action is its invariance under the constant translation of the scalar field, i.e., $\phi \rightarrow \phi+$ const which implies the existence of a conserved Noether charge. It is known that this hypothesis considerably simplifies the integration of the equations of motion. The action will contain up to second-order covariant derivatives of the scalar field and is parity invariant, that is invariant under the discrete transformation $\phi \rightarrow-\phi$. The action is parametrized in terms of six coupling functions that depend only on the kinetic term $X=g^{\mu \nu} \partial_{\mu} \phi \partial_{\nu} \phi$ of the scalar field. Recently it has been shown that such scalar tensor theories are invariant under a Kerr-Schild symmetry, and this symmetry turns out to be extremely useful for generating black hole solutions from simple seed configurations [17]. Here we will adopt a different strategy by deriving the most general stationary solution by brute force, as it was done for the special case of Horndeski theory in three dimensions [17]. Interestingly enough, we will show that the integration of the equations of motion forces the scalar field to have a constant kinetic term while at the same time the metric functions turn out to be a BTZ-like spacetime with an effective cosmological constant expressed in terms of the coupling functions appearing in the action. We would like to emphasize that the constant value of the kinetic scalar field term results from an algebraic equation that $X$ must satisfy, and consequently it does not correspond to any hair. Although the metric solution is given by a
BTZ-like metric, it is legitimate to wonder whether the presence of the scalar field could affect the thermodynamic properties of the solution. In order to answer this question, the thermodynamics of the solution is carefully analyzed within the Euclidean method $[18,19]$, and it is shown that the expressions of the mass, entropy and angular momentum are identical to those of a BTZ-like solution with an effective cosmological constant. In addition, since it has been pointed out that the Wald formula for the entropy [20] applied to general scalar tensor theories may be problematic [21], we have found it sensible to compute the entropy of the solution by means of a generalized Cardy formula. In this formulation the ground state is identified with a soliton whose mass is proportional to the lowest eigenvalues of the shifted Virasoro operators, see [22-26]. In order to achieve this task, we have constructed the static scalar soliton from the black hole through a double Wick rotation and computed its mass. Finally, the application of the generalized Cardy formula is shown to properly reproduce the semiclassical expression of the entropy.

The plan of the paper is organized as follows. In the next section we will present the action and derive the most general solution for a stationary ansatz for the metric together with a radial scalar field. We will show that the metric solution is nothing other than a BTZ-like metric while the kinetic term of the scalar field is constant. In Sec. III we will construct the regularized Euclidean action which allows us to identify the mass, the angular momentum and the entropy. Further, the expression of the entropy will be confirmed through a computation involving the generalized Cardy formula and the mass of the static scalar soliton. The mass of the soliton will be computed using the quasilocal formalism [27]. Finally, in Sec. IV we present our conclusions and discussions.

\section{SCALAR FIELD MODEL AND THE DERIVATION OF ITS SOLUTION}

In three dimensions, we are considering a scalar tensor theory whose dynamical fields are represented by a metric, $g$, and a scalar field, $\phi$. The action reads

$$
\begin{aligned}
S= & \int d^{3} x \sqrt{-g} \mathcal{L} \\
= & \int d^{3} x \sqrt{-g}\left[Z(X)+G(X) R+A_{3}(X) \square \phi \phi^{\mu} \phi_{\mu \nu} \phi^{\nu}\right. \\
& +A_{2}(X)\left((\square \phi)^{2}-\phi_{\mu \nu} \phi^{\mu \nu}\right)+A_{4}(X) \phi^{\mu} \phi_{\mu \nu} \phi^{\nu \rho} \phi_{\rho} \\
& \left.+A_{5}(X)\left(\phi^{\mu} \phi_{\mu \nu} \phi^{\nu}\right)^{2}\right],
\end{aligned}
$$

where for simplicity we have defined $X=\partial_{\mu} \phi \partial^{\mu} \phi$ and $\phi_{\mu \nu}=\nabla_{\mu} \nabla_{\nu} \phi$. Here, the six coupling functions $Z, G$ and $A_{i}$ for $i=2, \ldots 5$ are a priori arbitrary functions of the kinetic term $X$, and contain up to second-order covariant derivatives of the scalar field. It is easy to see that the action is 
invariant under the shift symmetry $\phi \rightarrow \phi+$ const, as well as under the discrete transformation $\phi \rightarrow-\phi$. The field equations of the action (1) are reported in the Appendix.

More general, scalar tensor theories can be considered with coupling functions that additionally depend on the scalar field, $Z=Z(\phi, X), G=G(\phi, X)$ and $A_{i}=A_{i}(\phi, X)$. It is interesting to note that for Lagrangians breaking the shift symmetry $\phi \rightarrow \phi+$ const, and of the form

$$
\mathcal{L}=Z(\phi)+G(\phi) R,
$$

there exist black hole solutions which drastically differ from the BTZ solution, see for example [28] and references therein. In contrast, as we will show below, for the shift symmetric action (1), the spectrum of black hole solutions is restricted to BTZ-like solutions.

We now look for black hole solutions of the action (1) with a stationary metric and a purely radial scalar field. The most general such ansatz can be parametrized as follows:

$$
\begin{aligned}
d s^{2} & =-f(r) d t^{2}+\frac{d r^{2}}{f(r)}+H^{2}(r)[d \theta-k(r) d t]^{2}, \\
\phi & =\phi(r) .
\end{aligned}
$$

After some tedious computations one can show that the field equations associated to the action (see the Appendix) will become fully integrable for the ansatz (2) by fixing the coupling function $A_{5}$ in terms of the others through the following relation:

$$
A_{5}=\frac{\left(2 A_{2}+X A_{3}+4 G_{X}\right)^{2}}{2 X\left(G+X A_{2}\right)}-\frac{A_{3}+A_{4}}{X},
$$

where $G_{X}=\frac{d G}{d X}$. This relation is quite similar to the fourdimensional DHOST conditions which ensure the absence of Ostrogradski ghosts $[14,15]$. Further, the emergence of the condition (3) is not surprising, since in the literature concerning scalar tensor theories of the type (1), most of the solutions are found for special relations between the coupling functions $Z, G$ and the $A_{i}$ 's, see e.g., [29-36].

In what follows, we will consider the action (1) with the coupling function $A_{5}$ given by the relation (3), and for later convenience, we also define the following expressions:

$$
\begin{gathered}
\mathcal{Z}_{1}=G+X A_{2}, \\
\mathcal{Z}_{2}=2 A_{2}+X A_{3}+4 G_{X} .
\end{gathered}
$$

We are now in the position to present the general derivation of the spinning solution. As a first step, we consider the following combination of the metric equations:

$$
\mathcal{E}_{t \theta}+k \mathcal{E}_{\theta \theta}=0,
$$

which yields a first integral given by

$$
\left(\mathcal{Z}_{1} H^{3} k^{\prime}\right)^{\prime}=0
$$

Further, the combination

$$
2 \sqrt{f^{3} X} \mathcal{E}_{r r}-J^{r}=0,
$$

where $J^{r}$ is the radial component of the conserved current (see the Appendix), permits to express the derivative of the metric function $f$ as

$$
f^{\prime}=-\frac{4 f H^{\prime} \mathcal{Z}_{1} \mathcal{Z}_{2} X^{\prime}+f H \mathcal{Z}_{2}^{2} X^{\prime 2}+4 H^{3} k^{\prime 2} \mathcal{Z}_{1}^{2}-8 H Z \mathcal{Z}_{1}}{8 H^{\prime} \mathcal{Z}_{1}^{2}+2 H \mathcal{Z}_{1} \mathcal{Z}_{2} X^{\prime}}
$$

Inserting this into the two combinations

$$
\begin{array}{r}
\frac{\mathcal{Z}_{2}}{f}\left(\mathcal{E}_{t t}+k \mathcal{E}_{t \theta}\right)=0, \\
\frac{\mathcal{Z}_{1}}{\sqrt{f X}} \mathcal{E}_{J}+\frac{\mathcal{Z}_{2}}{H^{2}} \mathcal{E}_{t \theta}=0,
\end{array}
$$

one obtains after some manipulations the following equation:

$$
k \mathcal{Z}_{2}\left(\mathcal{Z}_{1} H^{3} k^{\prime}\right)^{\prime}+4 H\left[\left(\mathcal{Z}_{1} Z\right)_{X}-Z \mathcal{Z}_{2}\right]=0
$$

By Eq. (5) the first term vanishes, leaving

$$
\left(\mathcal{Z}_{1} Z\right)_{X}-Z \mathcal{Z}_{2}=0
$$

It is easy to see that, for $Z=0$, Eq. (9) is completely degenerate and gives no information about the kinetic term. Consequently in what follows we impose $Z$ to be nonzero. Then the kinetic term $X$ must satisfy this algebraic equation which in turn implies that $X$ has to be constant. Moreover, we would like to stress that its constant value is not an integration constant but must be rather understood as follows: Given a scalar tensor theory (1)-(3) with specific coupling functions $Z, G$ and the $A_{i}$ 's, the constant value of $X$ will be determined by the algebraic relation (9). This restriction on the kinetic term of the scalar field significantly simplifies the equations, in particular the combination

$$
\frac{2}{f}\left(\mathcal{E}_{t t}+2 k \mathcal{E}_{t \theta}+k \mathcal{E}_{\theta \theta}\right)+2 f \mathcal{E}_{r r}+\sqrt{\frac{X}{f}} J^{r}=0
$$

implies

$$
H^{\prime \prime}=0 \text {. }
$$

Finally, after some redefinitions of the coordinates, the metric solution can be casted in a standard BTZ-like form as 


$$
\begin{gathered}
d s^{2}=-N(r)^{2} F(r) d t^{2}+\frac{d r^{2}}{F(r)}+r^{2}\left(d \theta+N^{\theta}(r) d t\right)^{2}, \\
N=1, \quad F=\left(\frac{Z}{2 \mathcal{Z}_{1}} r^{2}-M+\frac{J^{2}}{4 r^{2}}\right), \quad N^{\theta}=\frac{J}{2 r^{2}} .
\end{gathered}
$$

This metric is nothing but a BTZ-like solution with an effective cosmological cosmological constant given by $\Lambda_{\text {eff }}=-Z / 2 \mathcal{Z}_{1}$. Various comments can be made concerning the emergence of a BTZ-like metric solution together with a scalar field with constant kinetic term. First of all, it is remarkable that the equations of motion of the general class of scalar tensor theories, given by the action (1) together with the condition (3), are fully integrable and yield a BTZ-like solution. It is also remarkable that, although the model is defined in terms of the coupling functions $Z, G$ and $A_{2}, A_{3}$ and $A_{4}$, the resulting solution is shown to be parametrized in terms of $Z$ and through the combinations $\mathcal{Z}_{1}$ and $\mathcal{Z}_{2}$, as defined in Eq. (4). This would also imply that scalar tensor theories of the form (1)-(3) with different coupling functions $Z, G$ and $A_{i}$ 's can have the same effective cosmological constant $\Lambda_{\text {eff }}$, and hence they can be solved by the same BTZ-like metric. Moreover, it is easy to see that the action (1)-(3) enjoys a Kerr-Schild symmetry as defined in [17] whose implementation on the stationary ansatz (2) can be summarized as

$$
f(r) \rightarrow f(r)-a(r), \quad H(r) \rightarrow H(r), \quad k(r) \rightarrow k(r),
$$

with a constant mass term i.e., $a(r)=M$ which is a direct consequence of the kinetic term $X$ being constant. This can be put in analogy with the four-dimensional static case where solutions of the action (1) with constant kinetic term were shown to have the standard Coulomb mass term $a(r)=\frac{M}{r}$, see Ref. [17].

In summary, we have shown that for a stationary ansatz (2) the integration of the field equations of (1)-(3) forces $X$ to be constant together with a BTZ-like metric (12a) and (12b). In the following section, we analyze its thermodynamics.

\section{THERMODYNAMICS OF THE SPINNING SOLUTION}

The thermodynamics of the solution will now be determined by means of the Euclidean method [18,19], where the Euclidean continuation of the metric is obtained by setting $t=-i \tau$ in the ansatz (12a). In order for the resulting metric to be real, one can introduce a complex constant of integration for the Euclidean momentum as $J_{\text {Eucl }}=-i J$, where $J$ will be identified with the physical angular momentum. In order to avoid a conical singularity, the Euclidean time $\tau$ has to be made periodic with period $\beta=1 / T$, where $T$ is the temperature that in our case is given by

$$
T=\left.\frac{F^{\prime}(r)}{4 \pi}\right|_{r=r_{h}}=\frac{1}{4 \pi}\left(\frac{2 r_{h}}{L^{2}}-\frac{J^{2}}{2 r_{h}^{3}}\right),
$$

and where for simplicity we have defined the square of the effective AdS radius

$$
L^{2}=\frac{2 \mathcal{Z}_{1}(X)}{Z(X)} .
$$

Recall that the kinetic term $X$ is a constant determined by the algebraic relation defined by Eq. (9). After some computations, the Euclidean action is shown to be given by

$$
\begin{aligned}
I_{E}= & 2 \pi \beta \int_{r_{h}}^{+\infty} d r\left\{N \left[F^{\prime}\left(\frac{1}{4} r\left(F\left(\phi^{\prime}\right)^{2}\right)^{\prime} \mathcal{Z}_{2}+\mathcal{Z}_{1}\right)\right.\right. \\
& +\frac{1}{2} F\left(4\left(F\left(\phi^{\prime}\right)^{2}\right)^{\prime} \mathcal{Z}_{1 X}+r \mathcal{Z}_{2}\left(F\left(\phi^{\prime}\right)^{2}\right)^{\prime \prime}\right) \\
& +\frac{1}{4} F r\left(\left(F\left(\phi^{\prime}\right)^{2}\right)^{\prime}\right)^{2}\left(2 \mathcal{Z}_{2 X}-\frac{\mathcal{Z}_{2}^{2}}{2 \mathcal{Z}_{1}}\right)-Z r \\
& \left.\left.+\frac{1}{2} \frac{p^{2}}{\mathcal{Z}_{1} r^{3}}\right]+N^{\theta} p^{\prime}\right\}+B_{E},
\end{aligned}
$$

where $r_{h}$ is the radius of the event horizon and

$$
p(r)=\frac{r^{3}\left(N^{\theta}\right)^{\prime} \mathcal{Z}_{1}}{N} .
$$

The Euclidean action $I_{E}$ is defined up to a boundary term $B_{E}$ which is fixed such that said action has an extremum, which is $\delta I_{E}=0$. In the present case, the variation of this boundary term can be conveniently expressed as

$$
\begin{aligned}
\delta B_{E}= & -2 \pi \beta\left[\left(\left(\frac{\delta I_{E}}{\delta F^{\prime}}\right)-\left(\frac{\delta I_{E}}{\delta F^{\prime \prime}}\right)^{\prime}\right) \delta F\right. \\
& +\left(\frac{\delta I_{E}}{\delta F^{\prime \prime}}\right) \delta F^{\prime}+\left(\frac{\delta I_{E}}{\delta \phi^{\prime \prime}}\right) \delta \phi^{\prime}+\left(\frac{\delta I_{E}}{\delta \phi^{\prime \prime \prime}}\right) \delta \phi^{\prime \prime} \\
& -\left(\frac{\delta I_{E}}{\delta \phi^{\prime \prime \prime}}\right)^{\prime} \delta \phi^{\prime}+\left(\left(\frac{\delta I_{E}}{\delta \phi^{\prime}}\right)-\left(\frac{\delta I_{E}}{\delta \phi^{\prime \prime}}\right)^{\prime}\right. \\
& \left.\left.+\left(\frac{\delta I_{E}}{\delta \phi^{\prime \prime \prime}}\right)^{\prime \prime}+2 F \phi^{\prime}\left(\frac{\delta I_{E}}{\delta X}\right)\right) \delta \phi+N^{\theta} \delta p\right]_{r=r_{h}}^{r=+\infty} .
\end{aligned}
$$

At infinity, most of these terms cancel each other out, yielding

$$
\left.\delta B_{E}\right|_{+\infty}=\left.2 \pi \beta \mathcal{Z}_{1} \delta M \Rightarrow B_{E}\right|_{+\infty}=2 \pi \beta \mathcal{Z}_{1} M
$$

while that at the horizon

$$
\begin{aligned}
\left.\delta B_{E}\right|_{r_{h}} & =8 \mathcal{Z}_{1} \pi^{2} \delta r_{h}-2 \pi \beta \Omega \delta\left(\mathcal{Z}_{1} J\right) \Rightarrow \\
\left.B_{E}\right|_{r_{h}} & =8 \mathcal{Z}_{1} \pi^{2} r_{h}-2 \pi \beta \Omega \mathcal{Z}_{1} J .
\end{aligned}
$$

In this expression, $\Omega$ represents the chemical potential, defined by 


$$
\Omega=\lim _{r \rightarrow+\infty} N^{\theta}(r)-N^{\theta}\left(r_{h}\right)=-\frac{J}{2 r_{h}^{2}} .
$$

With all of the above, the boundary term $B_{E}$ is simply expressed as

$$
\begin{aligned}
B_{E} & =\left.B_{E}\right|_{+\infty}-\left.B_{E}\right|_{r_{h}} \\
& =2 \pi \beta \mathcal{Z}_{1} M-8 \mathcal{Z}_{1} \pi^{2} r_{h}+2 \pi \beta \Omega \mathcal{Z}_{1} J .
\end{aligned}
$$

Finally, the thermodynamic quantities can be read off from the Gibbs free energy $F$ :

$$
I_{E}=\beta F=\beta \mathcal{M}-\mathcal{S}-\beta \Omega \mathcal{J},
$$

where $\mathcal{M}$ is the mass, $\mathcal{S}$ the entropy and, as before, $\Omega$ is the chemical potential associated with the angular momentum $\mathcal{J}$, see [18]. Finally, comparing (15) with (16), the thermodynamic parameters turn out to be given by

$$
\begin{aligned}
& \mathcal{S}=8 \mathcal{Z}_{1} \pi^{2} r_{h}, \\
& \mathcal{M}=2 \pi \mathcal{Z}_{1} M=2 \pi \mathcal{Z}_{1}\left(\frac{r_{h}^{2}}{L^{2}}+\frac{J^{2}}{4 r_{h}^{2}}\right), \\
& \mathcal{J}=-2 \pi \mathcal{Z}_{1} J, \quad \Omega=-\frac{J}{2 r_{h}^{2}},
\end{aligned}
$$

and one can easily see that the first law holds, namely $d \mathcal{M}=T d \mathcal{S}+\Omega d \mathcal{J}$. These thermodynamic quantities (17) are identical to those of a BTZ-like solution with an effective AdS radius given by $L$. It is clear that in order to deal with positive mass (entropy) solutions, we have to impose that $\mathcal{Z}_{1}>0$ and $Z>0$. It is natural to compare the scalar tensor solutions with the standard BTZ solution which would correspond to setting $\phi=0$. In the static case, the difference between the free energies, $\Delta F$, of the BTZ solution and the scalar solution reads

$$
\Delta F=F_{\mathrm{BTZ}}-F=16 \pi^{3} T^{2}\left[\frac{\mathcal{Z}_{1}^{2}(X)}{Z(X)}-\frac{\mathcal{Z}_{1}^{2}(0)}{Z(0)}\right] .
$$

Hence, defining $P(X)=\frac{\mathcal{Z}_{1}^{2}(X)}{Z(X)}$, we conclude that for $P(X)<P(0)$ [respectively for $P(X)>P(0)$ ], the BTZ solution (respectively the scalar tensor solution) is thermodynamically favored.

We now proceed by rederiving the expression of the semiclassical entropy (17a) by means of a generalized Cardy formula. In this formulation, the entropy of the black hole solution can be microscopically computed provided the theory admits a regular scalar soliton which would be identified as the ground state of the theory, see [22,24]. In our case, the regular soliton will be obtained from the static black hole solution (12) with $J=0$ through a double Wick rotation $t \rightarrow i \theta$ and $\theta \rightarrow i t$ together with an identification for the location of the event horizon $r_{h}=L$ given by

$$
d s^{2}=-\frac{r^{2}}{L^{2}} d t^{2}+\left(\frac{r^{2}}{L^{2}}-1\right)^{-1} d r^{2}+\left(\frac{r^{2}}{L^{2}}-1\right) d \theta^{2},
$$

and the line element of the regular static scalar solution after a redefinition of the radial coordinate reads

$$
d s^{2}=-\cosh ^{2}(\rho) d t^{2}+L^{2} d \rho^{2}+\sinh ^{2}(\rho) d \theta^{2} .
$$

As done for example in Refs. [25,26], the mass of the soliton (19) will be computed within the quasilocal formalism defined in [27]. In order to be as self-contained as possible, we will elaborate the steps of the computations. To begin with, the variation of the action (1)-(3) can be schematically represented as

$$
\delta S=\sqrt{-g}\left[\varepsilon_{\mu \nu} \delta g^{\mu \nu}+\varepsilon_{(\phi)} \delta \phi\right]+\partial_{\mu} \Theta^{\mu}(\delta g, \delta \phi),
$$

where $\varepsilon_{\mu \nu}$ and $\varepsilon_{(\phi)}$ correspond to the equations of motions with respect to the metric $g_{\mu \nu}$ and the scalar field $\phi$ (see the Appendix), while $\Theta^{\mu}$ is a surface term whose expression is given by

$$
\begin{aligned}
\Theta^{\mu}= & \sqrt{-g}\left[2\left(P^{\mu(\alpha \beta) \gamma} \nabla_{\gamma} \delta g_{\alpha \beta}-\delta g_{\alpha \beta} \nabla_{\gamma} P^{\mu(\alpha \beta) \gamma}\right)\right. \\
& +\frac{\delta \mathcal{L}}{\delta\left(\phi_{\mu}\right)} \delta \phi-\nabla_{\nu}\left(\frac{\delta \mathcal{L}}{\delta\left(\phi_{\mu \nu}\right)}\right) \delta \phi+\frac{\delta \mathcal{L}}{\delta\left(\phi_{\mu \nu}\right)} \delta\left(\phi_{\nu}\right) \\
& -\frac{1}{2} \frac{\delta \mathcal{L}}{\delta\left(\phi_{\mu \sigma}\right)} \phi^{\sigma} \delta g_{\sigma \rho}-\frac{1}{2} \frac{\delta \mathcal{L}}{\delta\left(\phi_{\sigma \mu}\right)} \phi^{\sigma} \delta g_{\sigma \rho} \\
& \left.+\frac{1}{2} \frac{\delta \mathcal{L}}{\delta\left(\phi_{\sigma \rho}\right)} \phi^{\mu} \delta g_{\sigma \rho}\right],
\end{aligned}
$$

with $P^{\mu \nu \lambda \rho}=\delta \mathcal{L} / \delta R_{\mu \nu \lambda \rho}$, and $\mathcal{L}$ is the Lagrangian. Considering now the variation induced by a diffeomorphism generated by a Killing vector $\xi^{\mu}$ whose action on the metric and the scalar field read

$$
\begin{aligned}
\delta_{\xi} g_{\mu \nu} & =2 \nabla_{(\mu} \xi_{\nu)}, \quad \delta_{\xi} \phi=\xi^{\sigma}\left(\nabla_{\sigma} \phi\right), \\
\delta_{\xi}\left(\nabla_{\nu} \phi\right) & =\xi^{\sigma} \phi_{\sigma \nu}+\left(\nabla_{\nu} \xi^{\sigma}\right) \phi_{\sigma},
\end{aligned}
$$

we construct a Noether current given by

$$
\mathcal{L} \xi^{\mu}+2 \varepsilon^{\mu \nu} \xi_{\nu}-\Theta^{\mu}\left(\delta_{\xi} g, \delta_{\xi} \phi\right)=\nabla_{\nu} K^{\mu \nu},
$$

which is derived from the potential $K^{\mu \nu}$,

$$
\begin{aligned}
K^{\mu \nu}= & \sqrt{-g}\left[2 P^{\mu \nu \rho \sigma} \nabla_{\rho} \xi_{\sigma}-4 \xi_{\sigma} \nabla_{\rho} P^{\mu \nu \rho \sigma}+\frac{\delta \mathcal{L}}{\delta \phi_{\mu \sigma}} \phi^{\nu} \xi_{\sigma}\right. \\
& \left.-\frac{\delta \mathcal{L}}{\delta \phi_{\nu \sigma}} \phi^{\mu} \xi_{\sigma}\right] .
\end{aligned}
$$


As shown in [27], for each Killing field a corresponding conserved quantity can be constructed as

$$
Q(\xi)=\int_{\mathcal{B}} d x_{\mu \nu}\left(\delta K^{\mu \nu}(\xi)-2 \xi^{[\mu} \int_{0}^{1} d s \Theta^{\nu]}\right) .
$$

Here, $\delta K^{\mu \nu}(\xi)=K_{s=1}^{\mu \nu}(\xi)-K_{s=0}^{\mu \nu}(\xi)$ is the difference of the Noether potential interpolating between the solutions along the path parametrized by $s \in[0,1]$, and $d x_{\mu \nu}$ represents the integration over the two-dimensional boundary $\mathcal{B}$. For the Killing field, $\xi=\partial_{t}$, one obtains that

$$
\delta K^{r t}=-\frac{2 G}{L} \int_{0}^{1} d s \Theta^{r}=-\frac{\mathcal{Z}_{1}}{L}+\frac{2 G}{L},
$$

yielding a mass for the static soliton (19) given by

$$
\mathcal{M}_{\mathrm{sol}}=-2 \pi \mathcal{Z}_{1}
$$

which is negative by virtue of the fact that $\mathcal{Z}_{1}>0$ in order to ensure a positive mass and entropy of the black hole solutions. As is the case for the BTZ solution, there is a phase transition between the black hole and the soliton at the critical temperature

$$
T_{c}=\frac{\sqrt{2}}{4 \pi} \sqrt{\frac{Z(X)}{\mathcal{Z}_{1}(X)}} .
$$

We are now in position to provide a microscopic computation of the black hole entropy. As stressed in [22], the Cardy formula is more conveniently expressed in terms of the vacuum charge rather than the central charge:

$$
\mathcal{S}_{C}=4 \pi \sqrt{-\tilde{\Delta}_{0}^{+} \tilde{\Delta}^{+}}+4 \pi \sqrt{-\tilde{\Delta}_{0}^{-} \tilde{\Delta}^{-}},
$$

where $\left(\tilde{\Delta}_{0}^{ \pm}\right) \tilde{\Delta}^{ \pm}$are the (lowest) eigenvalues of the shifted Virasoro operators. The eigenvalues are related to the mass and angular momentum as [6]

$$
\mathcal{M}=\frac{1}{L}\left(\tilde{\Delta}^{+}+\tilde{\Delta}^{-}\right), \quad \mathcal{J}=\tilde{\Delta}^{+}-\tilde{\Delta}^{-} .
$$

On the other hand, since the scalar soliton is identified with the ground state of the theory, its mass (22) is proportional to the lowest eigenvalue

$$
\tilde{\Delta}_{0}^{ \pm}=\frac{L}{2} \mathcal{M}_{\mathrm{sol}} \cdot
$$

Finally, the Cardy formula (23) can be conveniently rewritten in terms of $\mathcal{M}, \mathcal{J}$ as

$\mathcal{S}_{C}=2 \pi \sqrt{-L \mathcal{M}_{\mathrm{sol}}(L \mathcal{M}+\mathcal{J})}+2 \pi \sqrt{-L \mathcal{M}_{\mathrm{sol}}(L \mathcal{M}-\mathcal{J})}$, and it can be verified that this correctly reproduces the semiclassical entropy (17), this is, $\mathcal{S}_{C}=\mathcal{S}$.

It is known from the pioneer work of Brown and Henneaux [5] that the asymptotic symmetries of the three-dimensional BTZ-like solution (12) consist in two copies of the Virasoro algebra with equal left and right moving central charges given by

$$
c_{+}=c_{-}=\frac{3 L(X)}{2 G_{N}},
$$

where $G_{N}$ is the Newton constant. Hence, according to the AdS/CFT correspondence this family of solutions (12) would correspond to a CFT with central charges given by (24) and depending explicitly on the constant value $X$ as determined by Eq. (9). This clearly emphasizes the difference with the BTZ solution in the sense that the scalar field, through its constant kinetic term $X$, will leave its mark. This would mean that, in principle, for a given CFT with equal central charges, it can be possible to adjust the form of $L(X)$ for its central charges to coincide with (24).

\section{CONCLUSIONS AND DISCUSSIONS}

In the present work, we have shown that the equations of motion of a very general class of scalar tensor theories (1)(3) can be fully integrated for a stationary metric ansatz together with a purely radial scalar field. Interestingly enough, the kinetic term of the scalar field solution was forced to be constant, while at the same time the spacetime metric resulted to be a BTZ-like metric with an effective cosmological constant expressed in terms of the coupling functions. It is somehow appealing that the spectrum of such general class of theories only consists of a BTZ-like metric with (different) effective cosmological constants. This observation is even more relevant considering that in four dimensions, theories which are much less general than that studied here admit black hole solutions that are asymptotically AdS, flat or even exhibit a rather exotic asymptotic behavior [29-36]. Even more, in four dimensions a recipe has even been given to construct black hole solutions from any simple seed metric [17]. Nevertheless, one can notice an important difference concerning the kinetic term of the scalar field solution between the threeand the four-dimensional situations. Indeed, solutions in four dimensions with a nonconstant kinetic term were shown to exist $[17,35]$, while in our case the algebraic relation (9) forces the kinetic term to be constant. One might also have thought that the presence of a coupled scalar field should have affected the thermodynamics of the solution but this was not the case. This is essentially due to the constancy of the kinetic term of the scalar field solution. It would be nice to provide a physical explanation for the emergence of a BTZ-like metric as the solution of such a very general class of scalar tensor theories (1)-(3). 
It is further intriguing that the equations of motion become fully integrable by imposing the condition (3) on the coupling function $A_{5}$. As mentioned before, this relation is quite similar to the four-dimensional DHOST conditions [14,15] which prevent the emergence of Ostrogradski ghosts. It would be compelling to explore this point more deeply. Moreover, it is worth mentioning that said BTZ-like solution remains a solution even if one replaces the scalar field ansatz with $\phi=q t+\psi(r)+L \theta$ in (2), and if $X$ still solves the algebraic Eq. (9). Note that in this case, the vanishing of the radial component of the current $J^{r}=0$ is a consequence of the field equation [37]. In [17] the uniqueness of this solution was shown for the quadratic Horndeski action. Whether or not it is unique in the general case has yet to be established.

In [17], it was shown that the solution generating method also applies for generalized Proca theories with solutions having a nonzero radial component for the potential [38]. Hence, in a complete analogy with the work done here, it will be interesting to look for black hole solutions in three dimensions for more general vector tensor theories [39].

\section{ACKNOWLEDGMENTS}

O. B. is funded by the Ph.D. scholarship of the University of Talca. M. H. gratefully acknowledges the kind support of the ECOSud Project No. C18U04. M. B. is supported by grant Programa Fondecyt de Iniciación en Investigación No. 11170037.

\section{APPENDIX: FIELD EQUATIONS ASSOCIATED TO THE ACTION (1)}

Here we report the equations of motion of the action (1) that are obtained by varying the action with respect to the metric $\mathcal{E}_{\mu \nu}$ and those with respect to the scalar field $\varepsilon_{(\phi)}$. The former are given by

$$
\mathcal{E}_{\mu \nu}:=\mathcal{G}_{\mu \nu}^{Z}+\mathcal{G}_{\mu \nu}^{G}+\sum_{i=2}^{5} \mathcal{G}_{\mu \nu}^{(i)}=0
$$

where

$$
\begin{aligned}
& \mathcal{G}_{\mu \nu}^{Z}=-\frac{1}{2} Z(X) g_{\mu \nu}+K_{X} \phi_{\mu} \phi_{\nu} \\
& \mathcal{G}_{\mu \nu}^{G}=G G_{\mu \nu}+G_{X} R \phi_{\mu} \phi_{\nu}-\nabla_{\nu} \nabla_{\mu} G+g_{\mu \nu} \nabla_{\lambda} \nabla^{\lambda} G \\
& \mathcal{G}_{\mu \nu}^{(2)}=-\phi_{\mu}\left(A_{2 X} \nabla_{\nu} X\right) \square \phi-\left(A_{2 X} \nabla_{\mu} X\right) \phi_{\nu} \square \phi-A_{2} \phi_{\nu \mu} \square \phi-\phi_{\nu \mu} \phi_{\lambda}\left(A_{2 X} \nabla^{\lambda} X\right)+\phi_{\nu} \phi_{\lambda \mu}\left(A_{2 X} \nabla^{\lambda} X\right)+\phi_{\mu} \phi_{\lambda \nu}\left(A_{2 X} \nabla^{\lambda} X\right) \\
& +A_{2} R_{\nu \lambda} \phi_{\mu} \phi^{\lambda}+A_{2} R_{\mu \lambda} \phi_{\nu} \phi^{\lambda}-A_{2} \phi_{\lambda \nu \mu} \phi^{\lambda}+\frac{1}{2} A_{2} g_{\mu \nu}(\square \phi)^{2}+g_{\mu \nu} \phi_{\lambda}\left(A_{2 X} \nabla^{\lambda} X\right) \square \phi+A_{2} g_{\mu \nu} \phi^{\lambda} \phi_{\rho \lambda}^{\rho}-A_{2} g_{\mu \nu} R_{\lambda \rho} \phi^{\lambda} \phi^{\rho} \\
& +\frac{1}{2} A_{2} g_{\mu \nu} \phi_{\rho \lambda} \phi^{\rho \lambda}+A_{2 X} \phi_{\mu} \phi_{\nu}\left((\square \phi)^{2}-\phi_{\lambda \rho} \phi^{\lambda \rho}\right), \\
& \mathcal{G}_{\mu \nu}^{(3)}=-\frac{1}{2} A_{3} \phi_{\mu} \phi_{\nu}(\square \phi)^{2}-\frac{1}{2} \phi_{\mu} \phi_{\nu} \phi_{\lambda}\left(A_{3 X} \nabla^{\lambda} X\right) \square \phi+\frac{1}{2} A_{3} \phi_{\mu} \phi_{\lambda \nu} \phi^{\lambda} \square \phi+\frac{1}{2} A_{3} \phi_{\nu} \phi_{\lambda \mu} \phi^{\lambda} \square \phi-\frac{1}{2} A_{3} \phi_{\mu} \phi_{\nu} \phi^{\lambda} \phi_{\rho \lambda}^{\rho} \\
& +\frac{1}{2} A_{3} R_{\lambda \rho} \phi_{\mu} \phi_{\nu} \phi^{\lambda} \phi^{\rho}-\frac{1}{2} \phi_{\mu}\left(A_{3 X} \nabla_{\nu} X\right) \phi^{\lambda} \phi_{\rho \lambda} \phi^{\rho}-\frac{1}{2}\left(A_{3 X} \nabla_{\mu} X\right) \phi_{\nu} \phi^{\lambda} \phi_{\rho \lambda} \phi^{\rho}-\frac{1}{2} A_{3} \phi_{\nu} \phi^{\lambda} \phi_{\rho \lambda \mu} \phi^{\rho}-\frac{1}{2} A_{3} \phi_{\mu} \phi^{\lambda} \phi_{\rho \lambda \nu} \phi^{\rho} \\
& -A_{3} \phi_{\nu} \phi^{\lambda} \phi_{\rho \lambda} \phi_{\mu}^{\rho}-A_{3} \phi_{\mu} \phi^{\lambda} \phi_{\rho \lambda} \phi_{\nu}^{\rho}+\frac{1}{2} g_{\mu \nu} \phi_{\lambda}\left(A_{3 X} \nabla^{\lambda} X\right) \phi^{\rho} \phi_{\sigma \rho} \phi^{\sigma}+\frac{1}{2} g_{\mu \nu} A_{3} \phi^{\lambda} \phi^{\rho} \phi_{\sigma \rho \lambda} \phi^{\sigma}+g_{\mu \nu} A_{3} \phi^{\lambda} \phi^{\rho} \phi_{\sigma \rho} \phi_{\lambda}^{\sigma} \\
& +A_{3 X} \phi_{\mu} \phi_{\nu}(\square \phi) \phi^{\rho} \phi_{\sigma \rho} \phi^{\sigma}, \\
& \mathcal{G}_{\mu \nu}^{(4)}=-A_{4} \phi_{\mu} \phi_{\nu} \phi^{\lambda} \phi_{\rho \lambda}^{\rho}+A_{4} \phi_{\lambda \mu} \phi^{\lambda} \phi_{\rho \nu} \phi^{\rho}-\phi_{\mu} \phi_{\nu}\left(A_{4 X} \nabla^{\lambda} X\right) \phi_{\rho \lambda} \phi^{\rho}-A_{4} \phi_{\mu} \phi_{\nu} \phi_{\rho \lambda} \phi^{\rho \lambda}-\frac{1}{2} A_{4} g_{\mu \nu} \phi^{\lambda} \phi^{\rho} \phi_{\sigma \rho} \phi_{\lambda}^{\sigma} \\
& +A_{4 X} \phi_{\mu} \phi_{\nu} \phi_{\lambda \rho} \phi^{\lambda} \phi^{\rho \sigma} \phi_{\sigma} \\
& \mathcal{G}_{\mu \nu}^{(5)}=-A_{5} \phi_{\mu} \phi_{\nu} \phi^{\lambda} \phi_{\rho \lambda} \phi^{\rho}(\square \phi)-\phi_{\mu} \phi_{\nu} \phi_{\lambda}\left(A_{5 X} \nabla^{\lambda} X\right) \phi^{\rho} \phi_{\sigma \rho} \phi^{\sigma}+A_{5} \phi_{\nu} \phi_{\lambda \mu} \phi^{\lambda} \phi^{\rho} \phi_{\sigma \rho} \phi^{\sigma}+A_{5} \phi_{\mu} \phi_{\lambda \nu} \phi^{\lambda} \phi^{\rho} \phi_{\sigma \rho} \phi^{\sigma} \\
& -A_{5} \phi_{\mu} \phi_{\nu} \phi^{\lambda} \phi^{\rho} \phi_{\sigma \rho \lambda} \phi^{\sigma}-2 A_{5} \phi_{\mu} \phi_{\nu} \phi^{\lambda} \phi^{\rho} \phi_{\sigma \rho} \phi_{\lambda}^{\sigma}-\frac{1}{2} A_{5} g_{\mu \nu} \phi^{\lambda} \phi_{\rho \lambda} \phi^{\rho} \phi^{\sigma} \phi_{\tau \sigma} \phi^{\tau}+A_{5 X} \phi_{\mu} \phi_{\nu} \phi^{\lambda} \phi^{\rho} \phi_{\rho \lambda} \phi^{\sigma} \phi^{\tau} \phi_{\tau \sigma} \text {, }
\end{aligned}
$$

while the field equations associated to the scalar field allow to construct a current conservation equation given by

$$
\varepsilon_{(\phi)}=\nabla_{\mu} J^{\mu}=\nabla_{\mu}\left[\frac{\delta \mathcal{L}}{\delta\left(\phi_{\mu}\right)}-\nabla_{\nu}\left(\frac{\delta \mathcal{L}}{\delta\left(\phi_{\mu \nu}\right)}\right)\right]=0,
$$

where 


$$
J^{\mu}=J_{Z}^{\mu}+J_{G}^{\mu}+\sum_{i=2}^{5} J_{(i)}^{\mu},
$$

with

$$
\begin{aligned}
J_{Z}^{\mu} & =2 Z_{X} \phi^{\mu}, \\
J_{G}^{\mu} & =2 G_{X} R \phi^{\mu}, \\
J_{(2)}^{\mu} & =2 A_{2 X} \phi^{\mu}\left[(\square \phi)^{2}-\phi_{\lambda \rho} \phi^{\lambda \rho}\right]-2 \nabla_{\nu}\left[A_{2}\left(g^{\mu \nu}-\phi^{\mu \nu}\right)\right], \\
J_{(3)}^{\mu} & =2 A_{3 X} \phi^{\mu} \square \phi \phi^{\lambda} \phi_{\lambda \rho} \phi^{\rho}+2 A_{3} \square \phi \phi_{\lambda}^{\mu} \phi^{\lambda}-\nabla_{\nu}\left[A_{3}\left(g^{\mu \nu} \phi^{\lambda} \phi_{\lambda \rho} \phi^{\rho}+\square \phi \phi^{\mu} \phi^{\nu}\right)\right], \\
J_{(4)}^{\mu} & =2 A_{4 X} \phi^{\mu} \phi^{\sigma} \phi_{\sigma \rho} \phi^{\rho \lambda} \phi_{\lambda}+A_{4}(X)\left[\phi_{\rho}^{\mu} \phi^{\rho \lambda} \phi_{\lambda}+\phi^{\sigma} \phi_{\sigma \rho} \phi^{\rho \mu}\right]-\nabla_{\nu}\left[A_{4}(X)\left(\phi^{\mu} \phi^{\nu \rho} \phi_{\rho}+\phi^{\sigma} \phi_{\sigma}^{\mu} \phi^{\nu}\right)\right], \\
J_{(5)}^{\mu} & =2 A_{5 X} \phi^{\mu}\left(\phi^{\sigma} \phi_{\sigma \rho} \phi^{\rho}\right)^{2}+2 A_{5}(X)\left(\phi^{\sigma} \phi_{\sigma \rho} \phi^{\rho}\right)\left(\phi^{\mu \sigma} \phi_{\sigma}+\phi^{\sigma \mu} \phi_{\sigma}\right)-2 \nabla_{\nu}\left[A_{5}(X) \phi^{\sigma} \phi_{\sigma \rho} \phi^{\rho} \phi^{\mu} \phi^{\nu}\right] .
\end{aligned}
$$

[1] M. Banados, C. Teitelboim, and J. Zanelli, Phys. Rev. Lett. 69, 1849 (1992).

[2] S. Deser, R. Jackiw, and S. Templeton, Phys. Rev. Lett. 48, 975 (1982); Ann. Phys. (N.Y.) 140, 372 (1982).

[3] E. A. Bergshoeff, O. Hohm, and P. K. Townsend, Phys. Rev. Lett. 102, 201301 (2009).

[4] J. M. Maldacena, Int. J. Theor. Phys. 38, 1113 (1999).

[5] J. D. Brown and M. Henneaux, Commun. Math. Phys. 104, 207 (1986).

[6] A. Strominger, J. High Energy Phys. 02 (1998) 009.

[7] S. Carlip, J. Phys. Conf. Ser. 67, 012022 (2007).

[8] E. Frodden, M. Geiller, K. Noui, and A. Perez, J. High Energy Phys. 05 (2013) 139.

[9] S. Hendi, B. Eslam Panah, and S. Panahiyan, J. High Energy Phys. 05 (2016) 029.

[10] R. Konoplya and A. Zhidenko, arXiv:2003.12171.

[11] K. Chan and R. B. Mann, Phys. Rev. D 50, 6385 (1994).

[12] S. Hossein Hendi, B. Eslam Panah, S. Panahiyan, and M. Hassaine, Phys. Rev. D 98, 084006 (2018).

[13] G. W. Horndeski, Int. J. Theor. Phys. 10, 363 (1974).

[14] H. Motohashi, K. Noui, T. Suyama, M. Yamaguchi, and D. Langlois, J. Cosmol. Astropart. Phys. 07 (2016) 033.

[15] J. Ben Achour, M. Crisostomi, K. Koyama, D. Langlois, K. Noui, and G. Tasinato, J. High Energy Phys. 12 (2016) 100.

[16] D. Langlois, R. Saito, D. Yamauchi, and K. Noui, Phys. Rev. D 97, 061501 (2018).

[17] E. Babichev, C. Charmousis, A. Cisterna, and M. Hassaine, J. Cosmol. Astropart. Phys. 06 (2020) 049.

[18] G. Gibbons and S. Hawking, Phys. Rev. D 15, 2752 (1977).

[19] T. Regge and C. Teitelboim, Ann. Phys. (N.Y.) 88, 286 (1974).

[20] R. M. Wald, Phys. Rev. D 48, R3427 (1993).

[21] X. Feng, H. Liu, H. Lü, and C. Pope, J. High Energy Phys. 11 (2015) 176.

[22] F. Correa, C. Martínez, and R. Troncoso, J. High Energy Phys. 01 (2011) 034.
[23] H. A. Gonzalez, D. Tempo, and R. Troncoso, J. High Energy Phys. 11 (2011) 066.

[24] F. Correa, A. Faundez, and C. Martínez, Phys. Rev. D 87, 027502 (2013).

[25] E. Ayon-Beato, M. Bravo-Gaete, F. Correa, M. Hassaine, and M. M. Juárez-Aubry, Phys. Rev. D 100, 044024 (2019).

[26] M. Bravo Gaete, L. Guajardo, and M. Hassaine, J. High Energy Phys. 04 (2017) 092.

[27] W. Kim, S. Kulkarni, and S.-H. Yi, Phys. Rev. Lett. 111, 081101 (2013).

[28] E. Ay'on-Beato, M. Hassaine, and J. A. Méndez-Zavaleta, Phys. Rev. D 92, 024048 (2015).

[29] E. Babichev, C. Charmousis, and A. Lehebel, J. Cosmol. Astropart. Phys. 04 (2017) 027.

[30] J. Chagoya and G. Tasinato, J. Cosmol. Astropart. Phys. 08 (2018) 006.

[31] E. Babichev, C. Charmousis, G. Esposito-Farse, and A. Lehbel, Phys. Rev. Lett. 120, 241101 (2018).

[32] T. Kobayashi and T. Hiramatsu, Phys. Rev. D 97, 104012 (2018).

[33] C. Charmousis, M. Crisostomi, R. Gregory, and N. Stergioulas, Phys. Rev. D 100, 084020 (2019).

[34] K. Takahashi, H. Motohashi, and M. Minamitsuji, Phys. Rev. D 100, 024041 (2019).

[35] M. Minamitsuji and J. Edholm, Phys. Rev. D 101, 044034 (2020).

[36] A. Lehébel, arXiv:1810.04434.

[37] E. Babichev, C. Charmousis, and M. Hassaine, J. Cosmol. Astropart. Phys. 05 (2015) 031.

[38] J. Chagoya, G. Niz, and G. Tasinato, Classical Quantum Gravity 33, 175007 (2016); M. Minamitsuji, Phys. Rev. D 94, 084039 (2016); E. Babichev, C. Charmousis, and M. Hassaine, J. High Energy Phys. 05 (2017) 114; A. Cisterna, M. Hassaine, J. Oliva, and M. Rinaldi, Phys. Rev. D 94, 104039 (2016).

[39] L. Heisenberg, R. Kase, M. Minamitsuji, and S. Tsujikawa, J. Cosmol. Astropart. Phys. 08 (2017) 024. 